\title{
Computer-aided Mandarin Test in Colleges: Problems and Solutions
}

\author{
Yuhan SONG \\ Chinese Department of the Engineering and Technical \\ College of Chengdu University of Technology, Leshan 614000, China \\ Slp609@163.com
}

Key Words: CAMT; Problems; Solutions

\begin{abstract}
Computer-aided Mandarin Test (CAMT) is a new window for Putonghua examination in China. As the use of modern information technology, CAMT brings a lot of advantages or merits for both testers and candidates. Meanwhile, it also brings some challenges to us. This paper will elaborates the main problems in CAMT and also gives some suggestive solutions to them in order to promote this novel type of language test.
\end{abstract}

\section{Introduction}

With the rapid development of computer and network, how to use information technology to solve problems has become a hot topic in the new century. Language testing is no exception. From the beginning of 1960s, computers are used to analyze tests, restore item banks and offer test results in a large scale. After 1980s, the traditional Paper-and-Pencil Based Language Testing (PBLT) has been replaced by Computer-based Language Test (CBLT) and further developed into Web-Based Individualized Self Adaptive Language Testing. For instance, in 2005 American Educational Testing Service (ETS) officially issued the rules of the online TOEFL measurement mode, that is, TOEFL iBT (Fulcher 2010). Therefore, the theory and practice of Computer-adaptive Language Testing are becoming a hot research topic in the testing mode of distance education.

In recent years, Computer-aided Mandarin Test (CAMT), as a new way of Putonghua test by modern technology, has also been popular in multiple cities in China. So far, CAMT has been carried out in 19 provinces with the help of the platform of the State Language Commission (SLC) and approximately 2 million people have taken part in the online test. CAMT to a certain degree is of great objective significance since it has changed the traditional paper-based and people-based test into a paperless and green activity. Although computers have brought some benefits for mandarin tests, some problems have also appeared with more and more college students pouring into them.

\section{Problems of CAMT}

\subsection{The Problems of the Testing System}

Firstly, although CAMT in colleges has been constantly upgraded many times and its interface is more and more simple, friendly and stable, there are some other problems in the practice, which need to be further solved, for example, the issue that the test software depends a lot on the hardware in the debugging process. At present, the equipment compatibility used for CAMT in colleges like sound card is very bad; hence, abnormal language recognition often occurs in testing process, thus affecting the normal work of the test. It is common knowledge that the maintenance rate of the electronic equipment, such as computers, is very low and therefore its replacement is very fast. So it 
is very urgent for colleges to purchase the newest hardware.

Secondly, the management interface function of the testing system is not perfect. For instance, there is no statistic function for the examination management. Therefore, some abnormal cases cannot be recorded timely, such as the absence of the students and the violation of the testing rules etc. Although these cases can be reported to the server of the provincial center after examination, it will take more labor and money. In addition, there is no examination instruction broadcast program for the monitoring to remind students of the operation rules and attentions. This problem is now solved by the examination management staff members, who will stress them orally many times before testing. It brings a lot of additional labor for the management workers and the effect is actually so-so.

Thirdly, the voice evaluation mode of the computer assessing system is poor (Liu Ai'min, 2011). At present, there is a uniform standard for CAMT in all colleges, that is, Outline of Putonghua Proficiency Test, in which a set of complete and standardized evaluations are listed in detail. Both candidates and academic research institutions can sum up the problems of voice and understand the details of scorers according to the scoring rules. However, the fact is that the actual score in the process is not fully implemented by this online although the testing software is introduced theoretically in terms of it.

\subsection{The Problems of the Management System}

For one thing, there are some loopholes in the information management of CAMT. In colleges and universities, the intelligent educational administration management system has been set up and used as various testing platforms, in which such students' information is included as name, gender, number, ID number, school, profession, class, and register time etc. However, it is very tedious and prone to error to operate in the information management system of CAMT because the students' information is not comprehensive in this system. For instance, there is no "class" information in it; therefore certificate management cannot be made by class as a management unit.

For another, random questions cannot be selected in present management system (Zeng Lin, 2012). Machine test software must be assigned tasks by test management staff members, so it will take a lot of time for them to change tasks for each time. If questions are changed for test of every time, the examination interval will become longer and its efficiency will become lower. In the practice of CAMT, students can get some examination content or information from each other because of such problems. Thus, the fairness of the test is lacked to a certain degree. What's more, because the testing tasks are allocated in a fixed machine, the examination for the second-time candidate who fails in the auditions must be taken in the original testing classroom. Thus ,the whole testing process will be influenced.

\subsection{The Problems in the Organization Process}

On the one hand, CAMT differs from the traditional manual test and it needs higher requirements for the staff members who must be familiar with the operation of the computers, learn the basic principles of the machine and be able to wipe out the errors in the testing process. However, the contradiction facts at present are: most of the front-line testers of CAMT cannot master the operation of the testing system while others with strong ability of computer operation are not good at speaking standardized mandarin Chinese. Especially for some older testers, their computer skill is not good, let alone their troubleshooting.

On the other hand, CAMT is a new test mode for the majority of candidates and there are some 
obstacles and adaptability for them psychologically and skillfully, so some targeted training should be given to the students before examination. In fact, due to the limitation of equipments, places and staff members etc, the real training is carried out in the form of meeting before test, focusing on the test processes and attentions. There are no chances for the candidates to operate on real computers, so they do not adapt to such kind of new test mode.

\section{Solutions of CAMT}

\subsection{To Optimize the Testing System}

Software performance is an important guarantee for successful CAMT. Its application is very wide, but its developing technology is very high. Therefore, the development and upgrading of the project is not enough if it relies on only a few bidding commissioned information technology companies, because the researcher are usually not the front-line testers of mandarin Chinese, the language teaching experts or theoretical researchers. So from this angle, the government should shoulder more responsibilities to make the research in this domain. It should especially absorb some researchers who are familiar with both computer technology and Putonghua test. In this way, the management, training and testing of CAMT can be modernized.

On the other hand, the development of any product cannot be completely perfect just one time; therefore, in the process of application, all colleges should find and record the relative problems, maintain close cooperation with the department of system development, and give timely feedback in the use of this technology. In this way, the testing system can be upgraded and optimized in time so as to further ensure the implementation of CAMT in colleges.

Besides, the network test environment should be carefully examined one week before the examination in order that the test can be carried out smoothly. At the same time, because the testing process should be recorded, the testing room should be a quiet place. When testing, it should be ensured that there are no large-scale activities or noises around the radio and the testing place.

\subsection{To Improve the Management System}

In terms of registration, generally speaking, there is students' information management system in colleges. Therefore, such kind of management system can be used as the platform to get the students' information. In this way, the staff members of the testing center can effectively avoid the manual entry of information and improve the speed of registration.

In terms of examination data, the examination management program can be compiled and used in the registration form to add key information such as students' ID, class number and telephone number and so on. Thus, it is easier for the staff members of the testing center to obtain the key information of the students. In addition, it is also important to solve the problem that a large amount of students take part in the examination, so it is better for students to register and download data online.

In terms of the supervision, the monitoring program and examination procedures can be designed to realize the following automatic operations: to send out the warning sound alarm when a test is abnormal, to give the entrance instruction to the candidates when a former test is over and to tell them attentions. As a result, the main job of the staff members in the testing center is not to supervise the candidates but to guide them to obey the testing disciplines. By the means of auxiliary examination procedures, CAMT becomes more automatic, smooth and orderly. Besides, such problem should also be solved that the staff members of the testing center cannot depart from the 
testing machine. Through these reforms, the standardization and automation of CAMT in China will gradually be realized.

\subsection{To Perfect the Organization Processes}

In order to perfect the organization processes, the following aspects should be stressed. In the first place, the staff training of the testing station is an important basis to ensure the standardization and normalization of the test. As the guider of CAMT, the staff members should be proficient in software installation and debugging, testing system maintenance and troubleshooting work etc. and be active in the innovation and optimization of the testing environment. Besides, they should be familiar with the whole processes of registration, verification, preparation, testing and evaluation in order to guide the candidates to successfully complete the test. In the second place, the college teachers of Mandarin Chinese should teach the students not only Putonghua itself but also some details about CAMT, such as computer-aided testing procedures, testing content, testing techniques and the psychological preparation so as to let them avoid fear, worry and anxiety. In the final place, special attentions should be paid to waiting room and preparing room. The waiting room should be kept a certain distance from the preparing room and the testing room so as to reduce the influence of external factors on the test. The preparing room should be closed to the testing room; thus it is more convenient for the candidate to enter the latter. In some cases, it should be considered that the waiting room and the preparing room can share the same machine room; thus the candidates can directly take part in the test after he or she selects the examination questions in order to ensure that he or she can have a better performance.

\section{Conclusion}

CAMT has been fully carried out and become a hot topic in Putonghua testing in China. It not only improves the testing efficiency but also reduces the workload of the staff members in the testing center. In practice, there will be some new problems which cannot be avoided. But it is believed that CAMT will have a better application and development if continuous improvement or constant innovation is made.

\section{References:}

[1] Fulcher, G. Practical Language Testing [M]. London: Hodder Education, 2010.

[2] Liu Ai'min. The Problems and Countermeasures of Computer-assisted Mandarin Test in Colleges [J]. Journal of Chongqing University of Arts and Sciences, 2011 (3): 90-92.

[3] Zeng Lin. Thoughts about Computer-aided Mandarin Test in Universities [J]. Journal of Sanming University , 2012 (3): 85-88. 